\title{
C. Didelon, C. Grasland, Y. Richard (dir.), Atlas de l'Europe dans le monde
}

Paris, Reclus/La Documentation française, coll. Dynamique du territoire, 2008, 260 p.

\section{Christian Vandermotten}

\section{(2) OpenEdition}

12 Journals

Édition électronique

URL : http://journals.openedition.org/belgeo/8104

DOI : $10.4000 /$ belgeo.8104

ISSN : 2294-9135

Éditeur :

National Committee of Geography of Belgium, Société Royale Belge de Géographie

Édition imprimée

Date de publication : 30 juin 2009

ISSN : 1377-2368

Référence électronique

Christian Vandermotten, "C. Didelon, C. Grasland, Y. Richard (dir.), Atlas de l'Europe dans le monde », Belgeo [En ligne], 2 I 2009, mis en ligne le 21 mai 2013, consulté le 22 septembre 2020. URL : http:// journals.openedition.org/belgeo/8104; DOI : https://doi.org/10.4000/belgeo.8104

Ce document a été généré automatiquement le 22 septembre 2020.

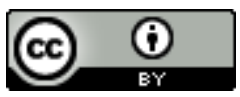

Belgeo est mis à disposition selon les termes de la licence Creative Commons Attribution 4.0 International. 


\title{
C. Didelon, C. Grasland, Y. Richard (dir.), Atlas de l'Europe dans le monde
}

Paris, Reclus/La Documentation française, coll. Dynamique du territoire, 2008, 260 p.

\author{
Christian Vandermotten
}

\section{RÉFÉRENCE}

C. Didelon, C. Grasland, Y. Richard (dir.), Atlas de l'Europe dans le monde, Paris, Reclus/La Documentation française, coll. Dynamique du territoire, 2008, 260 p.

1 Cet atlas poursuit la tradition des excellents travaux cartographiques commentés produits par le GDRE S4 du CNRS. Il s'agit ici de la publication de travaux qui s'inscrivent dans le programme ESPON d'observation du développement spatial et de l'aménagement du territoire européen.

2 Le thème examiné est celui de la place de l'Europe dans le monde. Il donne lieu a bien des réflexions originales, dont certaines se fondent sur des représentations cartographiques inattendues (parmi lesquelles on reconnaîtra évidemment l'appétence de Claude Grasland pour les représentations lissées, l'identification des discontinuités spatiales et le calcul de potentiels), des anamorphoses (selon le PIB, en parités de pouvoir d'achat, ou la population) ou encore sur le choix d'une projection polaire bien adaptée à rendre à la fois les relations entre pays de la Triade et les dépendances centre-périphérie.

3 L'ouvrage s'ouvre sur une réflexion sur le poids de l'Europe dans le monde (à quel(s) critère(s) recourir ?) et sur la (ou les) réalité(s) européenne(s). Pour ce qui est de l'Union européenne, elle maintient son poids dans le produit mondial, mais seulement à travers ses élargissements successifs - et pour combien de temps ? (p.75). Cela pose la question de nouveaux élargissements éventuels et donc des limites de l'Europe, des gradients de l'européanité, des contradictions entre perceptions culturelles, économiques et politiques, des illusions de continuité terrestres s'opposant aux mers 
perçues comme plus séparatrices. La vision des limites de l'Europe conduit à des configurations qui sont loin d'être uniformes, même parmi la communauté spécialisée des chercheurs en aménagement et en économie régionale rassemblée par ESPON (chapitre 4). Les cartes montrent aussi que la perception que les Européens ont "des autres" (p. 42) est sans doute influencée tout autant par l'importance économique ou politique que l'on accorde aux différentes portions du reste du monde que par leurs diversités objectives: pourquoi sinon, dans une partition du monde en 15 macrorégions proposées par les mêmes participants au réseau ESPON, l'Afrique est-elle considérée comme un ensemble unique, du Maroc à l'Afrique du sud, alors que l'Asie est fortement subdivisée (mais le Japon n'est curieusement pas séparé de la Chine ; eût-ce été le cas il y a vingt ans ?) et que l'Amérique centrale est séparée de celle du sud ? Les grands acteurs de la mondialisation et les entreprises transnationales organisent aussi le monde de manières diversifiées, où on reconnaît l'influence de leurs origines nationales (chapitre 3).

4 "Il n'y a de richesse que d'actifs" proclame le titre de la deuxième partie : c'est sans doute un slogan un peu abusif, qui néglige le qualitatif et n'est pas sans rappeler l'ultragauchisme de la révolution culturelle chinoise, qui remettait en question la politique anti-nataliste, au nom de la force attribuée au nombre. Il n'empêche que le renouvellement des actifs n'est plus assuré dans de nombreux pays membres de l'Union européenne : l'Allemagne, l'Italie et l'Espagne sont, avec le Japon, les pays où le rapport entre les âges jeunes et les âges actifs est le plus défavorable (p. 52). Les rapports de l'Europe avec "les autres" ne peuvent dès lors faire l'impasse sur la cartographie des différentiels démographiques: s'ajoutant aux déterminants culturels, ceux-ci expliquent sans doute pourquoi, vue par des Européens, la Méditerranée représente la dissociation subjective mondiale majeure entre ensembles territoriaux voisins (p.43). L'Europe tente plus de se prémunir des masses de population active disponibles sur le flanc sud de la Méditerranée que de s'y intéresser et de contribuer à leur formation. Pourtant, les gros effectifs de population active actuellement disponibles dans les nouveaux pays membres de l'Europe centre-orientale, qui donnent naissance à un important mouvement d'est en ouest, ne sont pas pour durer. Faut-il pour autant construire des indicateurs suggérant implicitement une "nécessaire" prolongation de la durée de la vie active, en fondant l'âge de l'arrêt du travail sur le nombre d'années restant à vivre (en bonne santé), comme cet indice de développement démographique durable, combinant l'âge médian et l'espérance de vie (chapitre 6) ? Une carte (p. 73) suggère en tout cas une Europe en "déclin doré", avec le Japon, c'est-à-dire assurant une croissance de sa richesse disponible par habitant au prix d'une baisse de sa part relative dans la population mondiale beaucoup plus rapide que celle de sa part dans le produit mondial, dans un contexte de population vieillissante. Mais ne seraient-ce pas plutôt, dans une perspective de développement durable, les critères mêmes des finalités de l'économie, et donc de la mesure de la croissance, qui devraient être envisagés?

5 La troisième partie de l'ouvrage illustre la position de l'Europe, avec celle des deux autres pôles de la Triade, au coeur de la mondialisation économique et financière, la Chine s'ajoutant à ces trois pôles majeurs si on considère aussi les échanges commerciaux. Différentes cartes examinent les asymétries structurelles, directionnelles et productives de ce commerce mondial, par rapport à l'Europe dans son ensemble, mais aussi en distinguant les spécificités nationales des pays européens. La carte de la p. 84 illustre bien la non-superposition entre l'espace des principaux 
partenaires commerciaux de l'Union européenne et celui des pays les plus tournés vers l'Europe, soit les pays africains, dont la place dans le commerce international se réduit malheureusement de plus en plus. Une série de graphiques examinent les soldes extérieurs du commerce européen avec le reste du monde par type de filière : le déficit énergétique est récurrent (le chapitre 14 y est plus loin consacré); celui dans l'agroalimentaire s'estompe; il apparait dans l'électronique depuis 1977 et dans le textile depuis 1987; l'Europe reste très excédentaire en mécanique et en chimie. L'Union européenne est aussi le premier émetteur mondial et le premier récepteur d'investissements directs à l'étranger (chapitre 9). Des cartes originales soulignent les spécificités de l'internationalisation des banques et des bourses européennes (chapitre 10).

6 Aucun aspect du positionnement de l'Europe (et de ses disparités nationales internes) n'est négligé par l'ouvrage. Ainsi, la quatrième partie situe l'Europe dans l'espace mondial des flux et des villes mondiales. L'information cartographique sur l'Europe aérienne et maritime est très riche. Le chapitre 15 traite des TIC. La cinquième partie est consacrée pèle-mêle aux droits sociaux et de l'homme, aux régimes alimentaires (la légende de la carte de la qualité de l'alimentation, p. 180, est partiellement inversée) : certes, les Européens mangent trop, mais ils échappent mieux à l'obésité que les NordAméricains (et aux maladies cardio-vasculaires que les Russes). L'atlas traite encore de l'environnement, du tourisme, du développement humain (de ce point de vue les évolutions entre 1975 et 2002 sont spectaculaires: le différentiel entre l'Union européenne et le versant sud de la Méditerranée s'est sensiblement réduit, alors que la situation relative s'est en revanche détériorée dans l'est européen non incorporé à l'Union), des proximités culturelles et historiques. Mais si l'Europe se projette vers le monde, avec une influence globale maximale en Afrique et en Russie (chapitre 22), elle tend aussi à s'en isoler : les cartes des morts à ses frontières (pp. 228-229) montrent une tendance au repli, comme une forteresse, alors pourtant que l'immigration vers l'Europe est moindre que celle vers les États-Unis, où la population est par ailleurs plus jeune (mais les demandeurs d'asile sont plus nombreux en Europe).

7 On comprendra de cette énumération non exhaustive que cet ouvrage original, dont on appréciera en particulier différentes cartes dont la légende est fondée sur la représentation graphique de typologies, est bien plus qu'un atlas sur la place de l'Europe dans le monde, mais vaut aussi comme une excellente cartographie commentée des conditions économiques, sociales, politiques, écologiques des pays du monde. 